\title{
A technique for producing highly complex visual stimuli*
}

\author{
WILLIAM H. WILSON and JUM C. NUNNALLY \\ Vanderbilt Lniversits, Vashille. Tennessee 37240
}

The production of randomly generated polygons of high complexity is a very time-consuming process with presently available methods. A technique was developed for speeding this process, particularly when producing very complex figures (e.g., polygons with over 40 sides). A portion of this process was computerized to facilitate production further. The procedure was employed to produce polygons with up to 200 sides. The technique proved feasible in practice, and subsequently clear results were obtained with the polygons in research on visual exploratory behavior.

Over the past 10 years, many studies have been concerned with the influence of various stimulus attributes on visual exploratory behavior. One stimulus attribute which has been examined frequently is complexity. Operationally, complexity has been defined in many ways, e.g., the random arrangement of dots in a given size matrix (Gamer \& Clement, 1963) or lights flashing in some random order (Unikel, 1971). Of course, stimulus complexity has frequently been defined in terms of the number of sides of a randomly generated polygon.

The most frequently employed techniques for generating random polygons were developed by Attneave and Arnoult (1956), who describe certain rules for connecting $x$ random points in an $n$ by $n$ matrix. These rules, when followed, yield a polygon with $\mathrm{x}$ or fewer sides.

In developing stimuli for research on visual exploratory behavior, the authors and their associates found it very difficult to employ Attneave's methods to produce polygons with high levels of complexity (e.g., polygons with more than 40 sides). A principle difficulty is the amount of time required to produce figures of about 40 turns and beyond. Also, these techniques are not sufficiently clear as to how one should proceed in producing very complex figures. Therefore, we developed procedures that might be more easily and efficiently applied when developing highly complex figures. A general method for this purpose is described as follows.

\section{METHOD}

There are five basic steps in the construction procedure.

(1) Randomly determine the coordinates for $x$ points and plot them in an $n$ by $n$ matrix. The points are numbered consecutively as they are plotted. Plotting may, of course, be done by hand using a table of random numbers. However, a

*The writing of this paper was supported, in part, by research grants from the National Science Foundation (No. GB29416) and from the United States Office of Education (No. OEG-4-71-0051) to J. C. Nunnally. The opinions expressed herein, however, do not necessarily reflect the position or policy of the U.S. Office of Education, and no official endorsement by the U.S. Office of Education should be inferred. simpler method is to have a computer randomly determine the coordinate pairs and plot them on a paper printout device. This is especially useful when $x$ becomes large (e.g. 300 points).

(2) Connect all of the outside points, forming a convex polygon, i.e., one with only convex angles. Outside points are those which when connected enclose all other points. This convex or Stage 1 polygon covers the maximum amount of area for the points plotted.

(3) Randomly select a line segment on the Stage 1 polygon as a starting point. Any of several methods for selecting this point is satisfactory. For example, one can select any point on the polygon and then count a random number of sides around the figure. The last side counted is then the starting point.

(4) Using a table of random numbers from 1 to $x$, select the first number which is not connected and draw lines from the two end points of the starting line segment to that number. This is the beginning of a Stage 2 polygon.

(5) Continue completely around the sides of the Stage 1 polygon, connecting (when possible) a point to each line segment as in Step 4 above.

Beginning with the construction of a Stage 2 polygon, there are four basic restrictions concerning which points may be connected to a line segment.

(1) If only one point can be connected to a line segment, that point is connected. If two or more points are available, a table of random numbers is used to select the point.

(2) No new line segment may be drawn that crosses a line segment of the figure under construction (Stage $k$ ) or the immediately preceding stage (Stage $\mathrm{k}-1$ ).

(3) Except in the Stage 1 polygon (i.e., convex), any point with a line through it that is not an end point of a line segment cannot be used as an end point until a later stage of the work. However, if three or more points fall in a straight line in the Stage 1 polygon, each point may be considered an end point.

(4) If a line segment cannot be connected to a point, that segment becomes part of the polygon currently being drawn.

The construction process can be envisioned as the incorporation of successive points into the various line segments of the Stage 1 polygon. Actually, what is done is to draw increasingly more complex polygons beginning with the convex polygon as Stage 1 and continuing to a Stage $\mathrm{N}$ where no more lines can be drawn.

The steps for producing a Stage 3 figure are exactly like those for the Stage 2, except that the lines of the Stage 2 polygon are followed. For example, in Fig. 1, the dotted lines represent the convex polygon (Stage 1) and the solid lines the second polygon (Stage 2). As can be seen, several points are not connected. These points can be incorporated into the figure by repeating Steps 3-5 and following the sides of the Stage 2 polygon. In constructing a Stage $\mathrm{k}$ polygon, one would follow the line segments of the Stage $k-1$ polygon.

The entire procedure is straightforward except for some needed discussion about two points. One of the time-consuming steps of the above procedure concerns the initial plotting of points. A computer can facilitate this process. The authors developed a simple FORTRAN IV program for this purpose. The program is initially given a vector of numbers from 1 to $\mathrm{x}$. This vector is then randomized twice: once for the $x$-axis elements of the coordinate pairs and once for the $y$-axis elements. The coordinates are then plotted by the computer, which also enumerates them as they are arranged in the vector. A single sheet of computer printer paper can serve as the matrix for plotting all points when a small number of points (e.g., 60 or less) are plotted. However, if a large number of points are to be plotted, then one can program the computer so that four consecutive sheets of paper serve as the matrix, i.e.. each sheet is one quadrant. These four sheets must then be alined. cut, and 


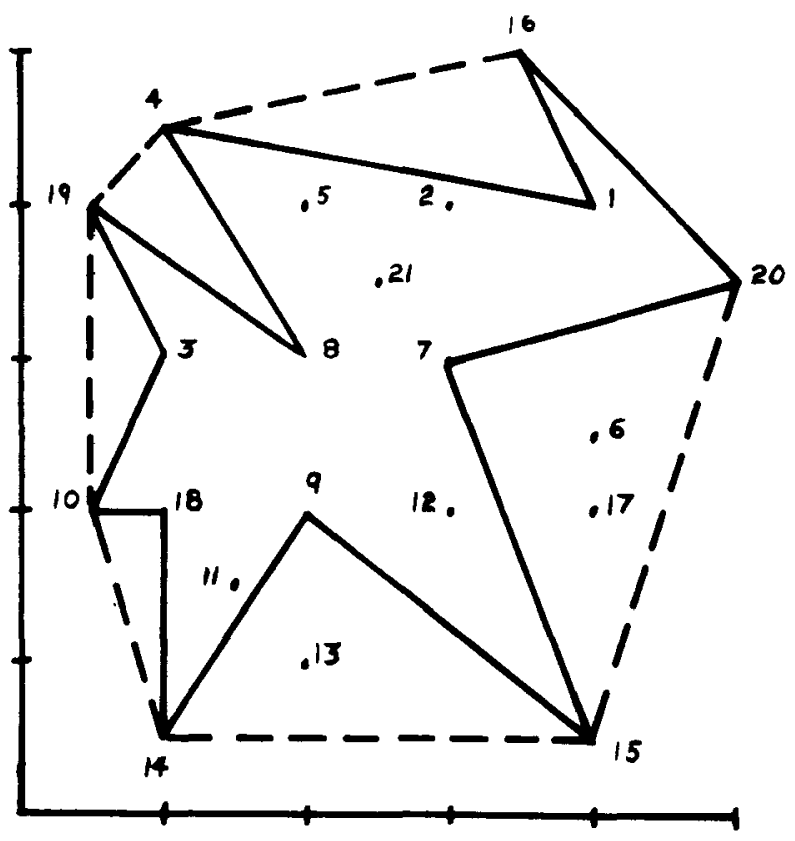

Fig. 1. First two stages in the construction of a random polygon.

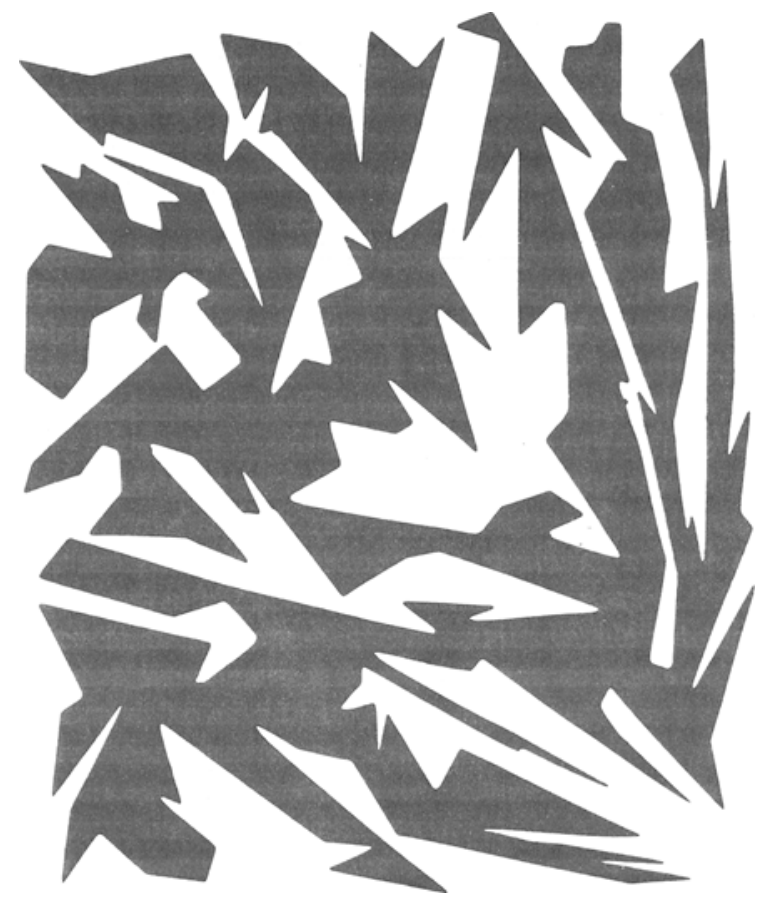

Fig. 2. A 200-sided polygon.

taped before drawing the figure. This latter procedure increases the plotted area sufficiently to facilitate drawing greatly. One can. of course. photographically reduce these initial plots to the desired size. Such a program greatly speeds the production of a large number of polygons, especially when each contains 200 to 300 points.

The second restriction is of principle importance in speeding the construction process; therefore, some further clarification may prove useful for its efficient use. When one has initially plotted a large number of points, even after several replications of Steps 3-5. there will be points which have not been connected. These points were not connected because: (1) they had not yet occurred in the table of random numbers, or (2) they did occur but could not be connected without crossing already existing line segments. Since the objective is to connect as many points as possible, $\mathrm{i}^{*}$ becomes desirable to permit certain lines to be crossed in order to make more points available to be connected. Lnder the second restriction, any line segment may be crossed which is not part of the stage being drawn or the immediately preceding stage.

An easy method of adhering to the second restriction is to employ a different color in drawing each of the successive stages. In this way, the lines which may be crossed are readily apparent even when the figure becomes very involved.

Another consideration which may not be immediately apparent is that no restrictions are placed on whether the point to be connected to a line segment lies inside or outside the existing figure (i.e., the last stage drawn). When drawing the Stage $k+1$ figure, one must follow the sides of the Stage $k$ figure. Hoving inside or outside the Stage $k$ figure at end points of each line segment does not constitute crossing a line segment of that figure. For example, in drawing a Stage 3 polygon in Fig. 1. the line segment 7-20 can be connected to Points 6 or 17 outside the Stage 2 figure or to Points 5, 2, or 21 inside the Stage 2 figure. This is particularly important since more points remain available to be connected.

\section{DISCUSSION}

The only apparent problem with the technique is the number of points which become unavailable at later stages of work. These points lie either too close to or on existing line segments. This problem is not great at lower levels of complexity (e.g.. polygons of 50 to 60 sides), but increases to a point of losing 30 or 40 points in a matrix of 300 points. This problem may be reduced by limiting the maximum length of the sides at Stages 2 and above.

There are numerous other restrictions one can employ to produce figures which have specific characteristics. For example. one may restrict the number of millimeters a point should be from an existing line before it is connected. since this may produce a figure which appears to be two figures connected at a point. One might also wish to place restrictions on the angular variability (e.g.. not drawing lines to a point if the angle formed is less than $10 \mathrm{deg}$ or greater than $170 \mathrm{deg}$ ). Such restrictions, of course. depend upon the needs of the individual investigation.

The technique as described above has been found to be efficient in developing polygons with up to 200 sides (see Fig. 2). The complexity of these figures as rated by Ss correlated .98 with the number of sides. Several of these highly complex figures were employed in a recent study of visual exploratory behavior. The results of this research demonstrate an increasing monotonic relationship between ho number of sides and length of viewing time (Lemond et al. 1972. Experiment III).

\section{REFERENCES}

Attneave, F., \& Amoult. M. D. The quantitative study of shape 
and pattern perception, Psychological Bulletin, 1956, 53. 452.471 .

Garner, W. R. \& clement, D. E Goodness of pattem and pattern uncertaint. Journal of Verbaí Learring \& Verbal Behavior, $1963,2,446-452$.

Lemond, L. C. Durham, R. L., Slater, G. P.. Wilson, W. H. \& Nunnally, $J$, $C$. The influence of varying degrees of complexity and sensory isolation on visual exploratory behavior. Privately circulated mantiseript, 1972, Department of Psychology, Vahderbilt University, available on request.

Unikel, I. P. Effects of changes in stimulation upon preference for stimulus complexity. Journal of Experimental Psycholosy, $1971,88,246,250$.

(Received for publication December 10. 1972: revision received January $27,1973$. ) 